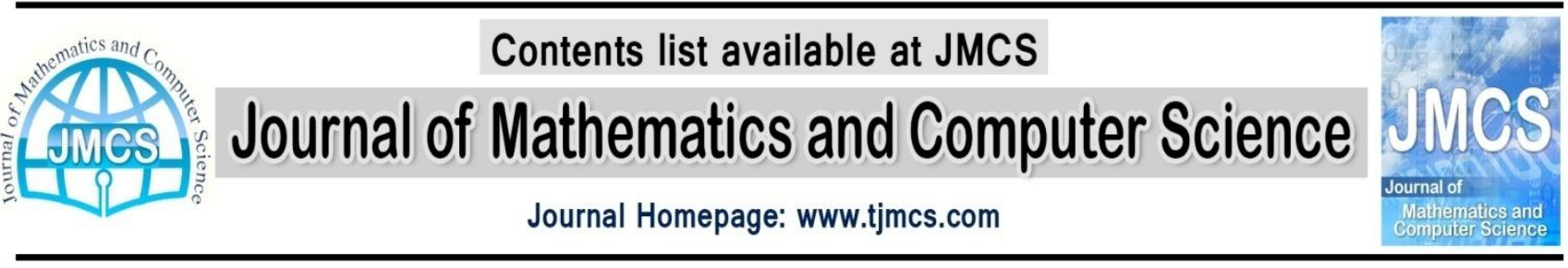

\title{
Designing and Implementing a Distributed Genetic Algorithm for Optimizing Work Modes in Wireless Sensor Network
}

\author{
Mehdi Eslami ${ }^{2}{ }^{*}$, Javad Vahidi², Majid Askarzadeh ${ }^{3}$ \\ ${ }^{1}$ Islaimic Azad University, Qeshm international branch, Qeshm, Iran, \\ ${ }^{2}$ Department of Applied Mathematics, Iran University of Science and Technology, Behshahr, Iran, \\ ${ }^{3}$ Islaimic Azad University, Saveh branch, Saveh, Iran, \\ *Eslaami.mehdi@gmail.com,jvahidi@iust.ac.ir,majid.askarzadeh@gmail.com
}

Article history:

Received May 2014

Accepted June 2014

Available online July 2014

\begin{abstract}
In this paper it is tried to present a solution for optimizing energy consumption in the sensors of wireless network by using distributed genetic algorithm and solving the famous problem of graph coloration. this idea formed by modeling sensors of wireless network by the help of graph and posing the problems of graph coloration with the description of work groups in scheduling nodes in wireless sensor networks. In this way we can save energy and conduct quality services in different time and place of wireless sensor network by determining some work groups in different time and different network tree node.
\end{abstract}

Keywords: Genetic Algorithm, Wireless Sensor Network, Graph Coloration, Optimizing Energy Consumption

\section{Introduction}

Nowadays, wireless sensor Network is widely being used in business, military, even researching areas and maintaining the natural sources. Although wireless sensor Network owns some qualities such as fast installation without having any initial scheme in each place and space, it has some shortcoming [1].

The biggest shortcomings this networks is their confined feeding sources of nodes (sensors), since dying the battery of a sensor makes it omitted from the Network.

preparing the work modes or making work shifting between the Networks sensors nodes is a method that would help the optimizing the energy consumption, and preventing the inactivation the sensors which have common nodes at the same times. 
One of the common plans for saving the energy source is performing the Modes in these networks. Since the wireless sensor Network categorization usually occurs randomly, a method should be wed to select the sensors working modes timing considering the sense field and their covering of sense field to level down the blind time and place nodes in Network.

One of the most practical and efficient Algorithm has been the Genetic Algorithm. This Algorithm has a great practicality and high potentials in solving the complicated problems which can solve many of them Genetic Algorithm is the one with a synthetic intelligence having some components:

1-1.population: In general, Initial population is a series of problems answers (chromosomes) which enter the algorithm firstly or be formed randomly [4]. The Initial population number depends on complicity and type of problem that can be identified by considering the number of answers which should be done parallely in each generation [5].

1-2.chromosome: chromosomes are formed by some genes that each gene is the sub-answer of the read one this answer can be proper, improper or even impossible. The Improper chromosomes are identified and would be considered as a less worthy ones and in next generation would be omitted or changed to the correct and logical anes (Figure 1).

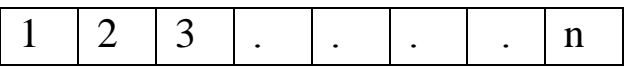

Fig. 1. Chromosome structure

1-3.encoding: encoding depends on the types of chromosome and changes depending what data each gene cam have [4]. For instance, we can encode all genes of a single chromosome as binary (zero \& one), or define as an integral number or according to the range in which a number can obtain.

In this article, coding is done as an integral number and each gene is considered as a work mode (color).

1-4.crossover: crossover in Genetic Algorithm plays an important role in convergency a reaching the optimum point that is done by single-point, two-point or multiple point methods as shown in figures 2 and 3 [4]. In this article one-point method came into use.

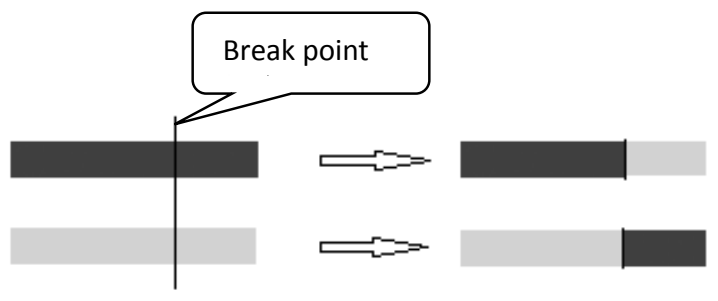

Fig. 2. Combination of single point

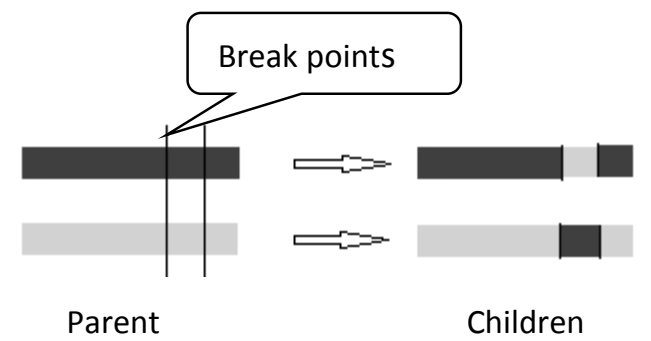

Fig. 3. The combination of the two-point 
1-5.mutation: random change of a gene in each bond is called mutation for example in coding, ones of the numbers in chromosome will be omitted and an other number in the span will be substituted.

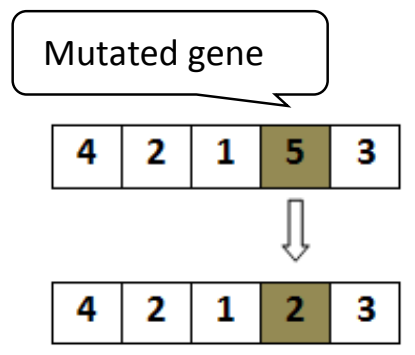

Fig. 4. Applying the mutation operator on a chromosome

1-6.selection: In selection phase, considering the suitability and conformity of the users expected conditions according to the defined cost of the function will receive a number suitability, then according to this suitability number that shows the properness of the considered answer will be selected by one the methods and afterwards will be introduced as the next parent- chromosome of the next generation.

A: leveling B: Rolt cycle. [4]

1-7.fitness: In actual is a factor that optimum making will be done according to it and shows the chromosome closeness and fitness, and conducting the algorithm is to this factor which is a phrase or program with some output that conduct the chromosomes to finding the best answer[2]. The main part in achieving the optimum answer in Genetic Algorithm method is the definition of the cored fitness, because if the conduct hadn't be done step-by-step, Algorithm can't get the proper answer or there will be needed to do move frequency repetition to get a better answer.

Modeling the sensor Network, we use a graph $G(\mathrm{~V}, \mathrm{E})$ that the sum of $v$ is the sum of centers in graph and $E$ is the sides of the graph. Since the different leveling of Networks nodes and their relations shows the graph and to make model of a complex network to do a simple task.

The Network in problem is considered as a graph.

1-8.Node: all wireless sensor distributed in the area is considered as a node.

1-9.Arc: Arcs in graph Networks are the connector between centers and having an arc between two nodes shows the relation between these nodes.

In graph coloration problem (GCP), the main point is finding the least number of colors for centers coloration G, In away that none of the two lateral centers heave the same color the least colors for this is called graph coloration number. This problem is one of the most complicated and NP-complete problems [2].

Johnson and other have shown that none of the final Algorithms were able to color the graph even with the small size of to centers [3]. Solving the Graph coloration problem is extremely hard and complicated one of the most important usage of Graph coloration is allocating registration in compilers and some timing problems and programming [1]. 
In this project we try to achieve a new way to timing the optimum modes of wireless sensor Network with the last deadend of time and place points. That have the ability of performing over all nodes and deviding the processed load over all Network nodes.

\section{LITERATURE REVIEW}

Protocol LEACH [6] is one of the primary orienting methods based on clustering in wireless sensor network .this protocol is composed of two starting and permanent phases a perform of these two phases is named round. In the first phase, the head clusters are selected, then the member of cluster will be selected. Sensor $n$ makes a random number between 0,1 ; if this number be less than of $t(n)$ in 1 , this sensor is selected as head cluster.

$$
T(n)= \begin{cases}\frac{p}{1-p \times\left(r \times \bmod \left(\frac{1}{p}\right)\right.} & \text { if } n \in G \\ 0 & \text { if } n \notin G\end{cases}
$$

In this equation, $\mathrm{p}$ is the optimum percentage of head clusters, $\mathrm{G}$ is the collection of nodes which were not selected as head cluster in $1 / P$, and $r$ is the number of the number of the round. When the head clusters selected, each of them send a message to introduce itself as a head cluster in network as the broadcast, and sensors select the most nearest head cluster, considering the received signal of head cluster; then performing a TDMA Scheduler program, each node will received a timing period for sending the received data. In phase 2 , the members of the cluster send the received data of the area to the head cluster according to the TDMA scheduler program. Before sending the data to the base station, the received signals will be processed to prevent the sending of overloaded data.

In [7], there is an algorithm named ECS that's about the improvement of LEACH method by changing the probability function. In this probability function, the energy parameter in clusters selection will be considered the defined probability function in this method is in form (2).

$$
P_{i}(r)=p \frac{E_{i}}{E_{\max }} \times\left[\frac{p \sqrt{r}}{N_{c h} \bmod 1 / p}+1\right]
$$

In this equation, $\mathrm{P}$ is the clustering rate, Emax is the primary energy of node $\mathrm{i}$, Ei is the remained energy of node $i$ and Nch is the times that node i selected as head cluster and $r$ is the number of round.

In algorithm LEACH [6], due to producing a random number, there is a probability of not selecting a single head cluster or a bunch of clusters. This causes the unfair energy consumption. Another problem in this method is the probability of selecting the all head clusters in a part of network that means there should be no proper distribution of head cluster. The ECS method was able to improve the protocol performance, but the above mentioned problems are still present in this method.

In [8], Gupta has used the fuzzy logic to find the head clusters. In this method, in each round of network the sensor that has the best chance of being a head cluster will be selected as the had cluster. To calculate the probability of chance of 3 fuzzy variable of the node energy, the Focus node (the number of nodes closed to a sensor) and Centrality node (showing the Centrality node) were used. In this method the base station is responsible of selecting the head clusters, do it selects the sensors data; then 
a sensor, that has the most chance according to the fuzzy rules, will be selected as head cluster. In this method, a head cluster will just be selected, a though there is a need to more head clusters to make the balance in energy consumption in network and increasing the network lie and this is necessary to consider the local information.

\section{Graph coloration with the goal of achieving the optimum modes}

In one Network sensor that is formed as a modeled graph, there is an arc between each two centers (sensor node) that are in radiation range of each other.

In this model we try to perform all centers to two or move working groups to some the energy by considering their working modes timing and take into account the service quality in sensor covering in time and place range of operation area.

In this model, sensor nodes are changed into different group to the graphs center coloration problem and to solve this problem, we use Genetic Algorithm.

sensors nodes placing in one group, are placed in one working mode in timing, so in order to maximizing the sensor coverage in each working mode and avoiding the coverage and qualifying the deadened point , It's better not to be in sensor field of each other.

In performed model, we used the graph coloration.

\section{Stages in performed program}

The structure of selecting problem (graph coloring) we want to color the graph of all nodes in a way that none of the lateral nodes have the same color and also to use the least needed colors.

The least color needed for this is called graph coloring number.

since the number of sensors considered for different goals are changeable and are choosed according the needs and wage and considering that nodes positions and their relation type will happen randomly while placing and their distribution, to enclose Its structure to a model and its read use, we try to make the user to consider their number depending on their needs and thoughts and their connections will be appear by one matrix with random arcs.

In other way, the main goal of this Article-optimizing the energy use with the help of performing the working modes between wireless Network nodes- is done by considering the number of colors in which the program is allowed to use them and user can determine it according to its needs in each time period.

\section{The structure of Genetic performer in G-coloring:}

\section{5-1. Encoding:}

In this problem, we use a factor as a chromo some factor indices as the mode- counter (sensors) are taken into account and this is convertible and will be placed in factors sectional places which are chosen randomly. In Initial coloring of chromosome, colors can be repetitive. In this factor colors are allocated randomly. In fact a loon matrix that is $\mathrm{n}$ number of our nodes and its amount is set by user which is colored randomly.

\section{5-2. Crossover:}

Considering that encoding will be done randomly the mixture type z-parents will be considered onepointed the factor randomly is chosen of the initial population that cut them from an open point 
randomly and change the numbers of cut area.

\section{5-3. Mutation:}

One gene is randomly selected and omitted and another random gene will be placed.

In this factor, that performs the gene mutation, considering the encoding randomly, mutation will be done by substitution in a way that one line and one raw will be chosen randomly from the initial population and a random number That is the color number will be placed there

\section{5-4. selection:}

The selection factor is an ascending code sorting that sort the initial population line based on their nt1 raw ascending that is called fitness.

\section{5-5. fitness:}

The neighboring nodes number, also with the same color inside the chromosome is considered as the assessment factor of it. It's definitely clear that the chromosome with lower fitness are more proper and better.

the assessment factor does the assessment for each initial population lines to make clear that for example Are the above- mentioned of $x$ and $y$ nodes in next matrix neighbor, it, yes they check the two nodes encoding to be with the same color. If yes, the add one two the fitness home of that chromosome (That is the performed matrix $n+1$ column).

The repetition of these steps continue when the program read the fitness of zero or the defined quantity. This operation in each node is done for some restricted chromosomes, then the optimum ones are sent to the manage-node and this manager node extracts the whole network optimum chromosomes after the result analysis and send them for next repetition to the nodes performing the performer Algorithm. There should be noticed that the nodes selected for distributed Algorithm be chosen and also have less working density and more energy saving.

\section{Discussion:}

Making these working modes in a wireless sensor network causes the saving in energy consumption of batteries sensors since the nodes will locate in a group in which they are not in a same sense range and this way sensory covering will lessen.

The possibility of making the service quality in this method is mentioned, because the more the group numbers in working nodes of the network the more energy will be saved. But the possibility of making the deadend sensory points will also increase in another words as much as the number of nodes in working nodes timing decreases, the energy use will more and the network sight ability will be better. So it is possible to adjust and improve the service quality based on environmental changes and wage needs by reperforming of the nodes with this method. for example the Network different activity at day and night and change of working mode in sensitive working times, also the intelligent adjustment of service quality in more sensitive place of network will be provided by choosing the colors number at working modes timing and different adjusting of working mode in different time and places.

Figure 5 shows a network that is connected to a graph. The connections are created randomly in this network. Now we describe an example of Algorithm performance.

It is to be considered that we had made two methods for sensors working made; the active mode when the sensor node is active and sleep mode when the sensor is not active. 


\begin{tabular}{|c|c|c|c|c|c|}
\hline & 1 & 2 & 3 & 4 & 5 \\
\hline 1 & 0 & 1 & 0 & 1 & 1 \\
\hline 2 & 1 & 0 & 0 & 0 & 1 \\
\hline 3 & 0 & 0 & 0 & 1 & 1 \\
\hline 4 & 1 & 0 & 1 & 0 & 0 \\
\hline 5 & 1 & 1 & 1 & 0 & 0 \\
\hline
\end{tabular}

Fig 5. Adjacency matrix of a network graph

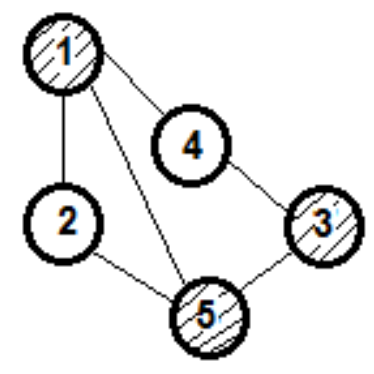

Fig 6. Timing modes for the two groups working

In Figure 6 the two groups in the network is used for timing and modes of work are as follows:

\begin{tabular}{|c|c|c|c|}
\hline $\begin{array}{l}\text { Working } \\
\text { mode }\end{array}$ & \multicolumn{3}{|c|}{ Sensor nodes in each group } \\
\hline 1 & 1 & 3 & 5 \\
\hline 2 & 2 & 4 & - \\
\hline
\end{tabular}

Table 1. Network nodes in two working groups

In working mode of 1 , sensors 1,3 and 5 are active sensor 1 and 3 and 5 are guarding instead of $(2,, 4 \& 5),(4 \& 5)$ and $(1,2 \& 3)$, respectively and sensor 2 and 4 for sensors (1\&5)and (1\&3) respectively.

If we pay attention more, we will understand that there is no deadend point in the network but for some sensors covering. That are in a same sense range and are active at the same time, the network will tolerate more energy use. 


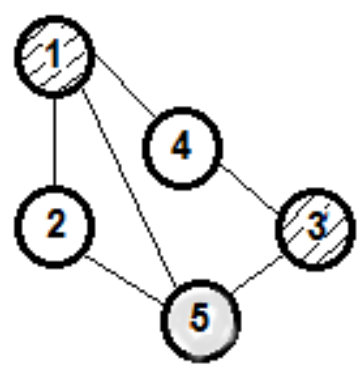

Fig. 7. Network nodes in three working groups

In Figure 7 the three working modes for timing sensor network that is used is as follows:

\begin{tabular}{|c|c|c|}
\hline $\begin{array}{l}\text { Working } \\
\text { mode }\end{array}$ & \multicolumn{2}{|c|}{ Sensor nodes in each group } \\
\hline 1 & 5 & - \\
\hline 2 & 1 & 3 \\
\hline 3 & 2 & 4 \\
\hline
\end{tabular}

Table 2. Network nodes in three working groups

sensor 5 is active in working mode 1 , thanks to be in a same sensory range with sensors $(1,2 \& 3)$, sensor 5 is guarding for them and these is no sensory point covering, but because It can't control the sensor 4 area, there would be a dead end point in network. In working mode 2 sensors $1 \& 3$ are active that sensor 1 and 3 are guarding instead of $(2,4, \& 5)$ and $(4 \& 5)$ respectively and in mode 3 sensors $2 \& 4$ are active that sensor 2 and 4 are guarding instead of $(1 \& 5)$ and $(1 \& 3)$ respectively.

We will understand that due to the few covering of sensors of same group, the energy consumption will also decrease to the less but there is still a deadend place in it.

\section{Conclusion}

With regard to the information described about the wireless sensor network we can say that the role of energy is the most important issue in these networks and with regard to small size and the amount of sensor and energy resources we should have special attention to them. Since the amount of sensor can be a lot in a wireless sensor network, we decided find an efficient way in scheduling optimized work models in wireless sensor network with the help of distributed genetic algorithm that is a smart algorithm with high capability in optimization by the aim of conducting suitable service quality and determining the amount of effective activity of nodes and saving energy sources.

\section{References}

[1] A.C.M. Ran, M.C.B. Reurings, A fixed point theorem in partially ordered sets and some applications to matrix equations, Proc. Amer. Math. Soc. 132 (2003) 1435-1443.

[1] I. Akyildiz, W. Su, Y. Sankarasubramaniam, E. Cayirci, A Survey on Sensor Networks, IEEE Communications Magazine, Vol. 40, No. 8, pp. 102-116, August 2002 
[2] G.J. Chaitin, Register Allocation and splitting via graph coloring, Proc. Of ACM SIGPLAN 82 Symposium on Compiler Construction, pp. 98-105, NewYork, 1987.

[3] D.S. Jobnson, C.R. Aragon, L.A. McGoach, C. Schevon, Optimization by simulated annealing: an exprimental evaluation. Part 2, graph coloring and number partitioing, Operations Research, vol. 39(3), pp. 378-406, 1991.

[4] David E. Golldberg, "Genetic Algorithms in Search, Optimization and Machine Learning", AddisonWesly Pub., 1989.

[5] Su-Young Parka, Jung Hyun Choia, Sookyun Wangb, Seok Soon Parka, Design of a water quality monitoring network in a large river system using the genetic, algorithm, Ecological Modelling 199 , pp 289-297, 2006.

[6] W. Heinzelman, A. Chandrakasan and H. Balakrishnan, Energy-Efficient Communication Protocol for Wireless Microsensor Networks, In Proceedings of the $33^{\text {rd }}$ Hawaii International Conference on System Sciences (HICSS '00), January 2000.

[7] I. Sim, K. Jin Choi, K. Kwon and Jaiyong Lee, Energy Efficient Cluster header Selection Algorithm in WSN", In Proceedings of IEEE International Conference on Complex, Intelligent and Software Intensive Systems, pages 584-587, March 2009.

[8] Gupta, D. Riordan and S. Sampalli, Cluster-head Election using Fuzzy Logic for Wireless Sensor Networks, In Proceedings of IEEE Communication Networks and Services Research Conference, pages 255- 260, May 2005. 